\title{
Exercise capacity of adolescent girls with idiopathic scoliosis; analyzed in 6 Minute Walking Test (6MWT), with and without Chêneau's brace - pilot studies
}

\author{
J Pajak, J Durmala, J Bugala-Szpak \\ From 9th International Conference on Conservative Management of Spinal Deformities - SOSORT 2012 \\ Annual Meeting \\ Milan, Italy. 10-12 May 2012
}

\section{Aim}

Establishing if Chêneau's brace decreases exercise capacity in adolescent patients with idiopathic scoliosis.

\section{Methods}

This is a prospective, randomized study. The research was done in 22 girls (mean age $15 \pm 1.7$ y.) with DM idiopathic scoliosis (mean Cobb angle - 33 degrees). These patients were qualified to conservative treatment using Chêneau's brace. Every studied person was acquainted with the technique of carrying out 6MWT, and realized the tentative test before proper exercise tests in the aim of the elimination of "the learning's effect". Tests were executed twice, in the brace and without the brace. The order or carrying out each test was random, so that half of the patients executed their first exercise test in the brace and the other half took their first test without the brace.(two various balls were used for the drawing).

\section{Results}

There was a statistically significant longer distance for the walking test, by patients without Chêneau's brace, in comparison to these same patients with Chêneau's brace. $(676.2 \pm 61.26 \mathrm{~m}$ vs. $631.8 \pm 78.31 \mathrm{~m})$ A positive correlation (non significant) was observed among the length of the distance in 6MWT and the size of kyphosis.

\section{Conclusions}

This initial study showed;
1. Chêneau's brace essentially diminishes exercise capacity for patients with idiopathic adolescent scoliosis.

2. A higher value of the kyphosis angle can reduce the negative influence of Chêneau's brace on exercise capacity.

Published: 3 June 2013

References

1. Katsaris G, Loukos A, Valavanis J, Vassiliou M, Behrakis PK: The immediate effect of a Boston brace on lung volumes and pulmonary compliance in mild adolescent idiopathic scoliosis. Eur Spine J 1999, 8(1):2-7.

2. DiRocco PJ, Breed AL, Carlin Jl, Reddan WG: Physical work capacity in adolescent patients with mild idiopathic scoliosis. Arch Phys Med Rehabil 1983, 64(10):476-478

3. Refsum HE, Naess-Andresen CF, Lange JE: Pulmonary function and gas exchange at rest and exercise in adolescent girls with mild idiopathic scoliosis during treatment with Boston thoracic brace. Spine 1990, 15(5):420-423.

doi:10.1186/1748-7161-8-S1-018

Cite this article as: Pajak et al:: Exercise capacity of adolescent girls with idiopathic scoliosis; analyzed in 6 Minute Walking Test (6MWT), with and without Chêneau's brace - pilot studies. Scoliosis 2013 8(Suppl 1): 